\title{
Overview of the italian experience in surgical management of bladder cancer during first month of COVID-19 pandemic
}

\author{
Carmen Maccagnano ${ }^{1}$, Lorenzo Rocchini ${ }^{2}$, Emanuele Montanari ${ }^{2}$, Giario Natale Conti ${ }^{1}$, \\ Giovanni Petralia ${ }^{3}$, Federico Dehò ${ }^{4}$, Kadi-Ann Bryan ${ }^{5}$, Roberto Contieri ${ }^{6}$, Rodolfo Hurle ${ }^{6}$ \\ ${ }^{1}$ Department of Surgery, Section of Urology, ASST Lariana, Nuovo Ospedale Sant'Anna, San Fermo della Battaglia (CO), Italy; \\ ${ }^{2}$ Department of Surgery, Division of Urology, Fondazione IRCCS Ca' Granda Ospedale Maggiore Policlinico, Milan, Italy; \\ ${ }^{3}$ Urology Unit, Niguarda Hospital, Milan, Italy: \\ ${ }^{4}$ Unit of Urology, ASST Sette Laghi-Circolo e Fondazione Macchi Hospital; \\ ${ }^{5}$ Rogue-Valley Urology, Medford, Oregon, US; \\ ${ }^{6}$ Department of Urology, Istituto Clinico Humanitas IRCCS Clinical and Research Hospital, Rozzano (MI), Italy.
}

\section{Summary Objective: Overview of bladder cancer $(B C)$ management in Italy during the first month} of the COVID-19 pandemic (March 2020) with head to head comparison of the data from March 2019, considered "usual activity" period. The aim is to analyze performance of different Italian Centers in North, Center and South, with a special eye for Lombardy (the Italian epicenter).

Patients and methods: During April 2020, a survey containing 14 multiple-choice questions focused on general staffing and surgical activity related to BC during the months of March 2019 and March 2020 was sent to 32 Italian Centers.

Statistical analysis was performed using IBM SPSS Statistics (v26) software. A Medline search was performed, in order to attempt a comparative analysis with published papers.

Results: 28 Centers answered, for a response rate of $87.5 \%$. Most of the urology staff in the Lombardy region were employed in COVID wards ( $p=0.003)$, with a statistically significant reduction in the number of radical cystectomies $(R C)$ performed during that time ( $p=0.036$ ). The total amount of RC across Italy remained the same between 2019 and 2020, however there was an increase in the number of surgeries performed in the Southern region. This was most likely due to travel restrictions limiting travel the North. The number of Trans-Urethral Resection of Bladder Tumors (TURBT) ( $p=0.046)$ was higher in Academic Centers (AC) in 2020 $(p=0.037)$.

Conclusions: The data of our survey, although limited, represents a snap shot of the management of $B C$ during the first month of the COVD-19 pandemic, which posed a major challenge for cancer centers seeking to provide care during an extremely dynamic clinical and political situation which requires maximum flexibility to be appropriately managed.

KEY WORDS: COVID-19; Urology; Pandemic; Outbreak; Bladder cancer; Non-muscle invasive bladder cancer; Trans-urethral resection of bladder tumor; Cystectomy; Hematuria.

Submitted 2 June 2020; Accepted 20 July 2020

\section{INTRODUCTION}

Bladder cancer (BC) represents 3\% of all malignancies, with 549.000 new cases and 199.000 deaths were reported worldwide in 2018 (1). This is generally associated with high levels of morbidity and mortality especially in patients over 70yrs. In the United States about $47 \%$ are estimated to be $\mathrm{Ta} / \mathrm{Tis}$ at initial presentation, $21 \%$ stage I, $11 \%$ stage II, $4 \%$ stage III, and $6 \%$ stage IV disease (2). The cornerstones of BC surgical treatments are represented by trans-urethral resection of bladder tumours (TURBT) and radical cystectomy (RC).

Some papers were recently published regarding mortality risk from COVID-19 and BC: 63\% of patients (pts) had one comorbidity (such as hypertension, cardiovascular, or pulmonary), $32 \%$ had two or more comorbidities, and the risks of dying from $\mathrm{BC}$ or from a competing disease were similar at 5 yrs after diagnosis $(3,4)$.

On March $11^{\text {th }}, 2020$, after the World Health Organization declared the disease caused by the novel Coronavirus SARS-COV-2 a pandemic, the exponential increase in the number of affected individuals led to a rapid reallocation of economic, infrastructural and health care resources, with redistribution of those medical/surgical, including urologic oncology, prioritizing urgent and emergent needs (5). This takes into consideration the evidence that almost 30\% of individuals older than 65 years are at risk for developing acute respiratory distress syndrome after contracting COVID-19 and approximately 20\% of asymptomatic individuals infected with COVID-19 may die after an elective operation $(6,7)$. However, the effect of this prioritization, as well as the clinical consequences of postponing surgical procedures, on patients and health care systems remains actively debated, despite recently published recommendations (7-12) (Table 1). Additionally, as demonstrated by Liang et al. and Moschovas et al., the increasing risk of COVID-19 related complications in cancer patients has to be considered specifically, noting that a significantly higher proportion of patients will require ventilation in the Intensive Care Unit (ICU), with some dying as a consequence of these complications $(13,14)$.

Moreover, 25\% of pts requiring high-priority surgery are considered at increased perioperative risk, which can be partially defined by cancer-related immunocompromised state and consequent higher susceptibility to 


\section{Table 1.}

International Scientific Societies recommendations about surgical management of BC during pandemic.

\begin{tabular}{|c|c|c|}
\hline Scientific Society & TURBT & Cystectomy \\
\hline $\begin{array}{l}\text { Italian Society of Urology (SIU) } \\
\text { (https://www.siu.it) (9) }\end{array}$ & $\begin{array}{l}\text { Low priority (deferrable) } \\
\text { - TURBT after instillations } \\
\text { - TURBT in low risk pts for progression } \\
\text { Intermediate priority } \\
\text { TURBT in pts with small recurrences } \\
\text { High priority: } \\
\cdot \text { Pts with high risk of progression } \\
\cdot 2^{\text {nd }} \text { look TURBT for HG or T1 }\end{array}$ & Never postpone \\
\hline $\begin{array}{l}\text { European Association of Urology (EAU) } \\
\text { (https://uroweb.org/wp-content/uploads/EAU- } \\
\text { Guidelines-Office-Rapid-Reaction-Group-An-organisation- } \\
\text { wide-collaborative-effort-to-adapt-the-EAU-guidelines- } \\
\text { recommendations-to-the-COVID-19-era.pdf) (10) }\end{array}$ & 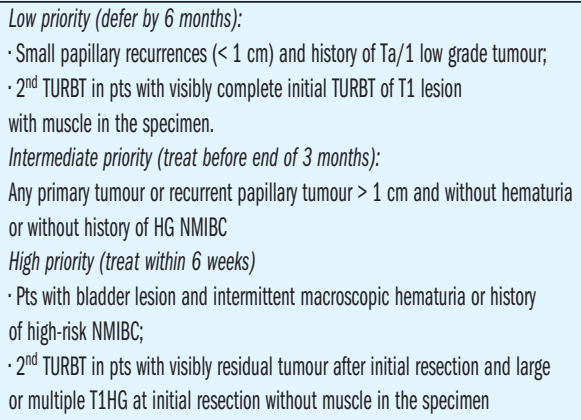 & $\begin{array}{l}\text { Cystectomy has to be performed within } 3 \text { months since } \\
\text { the diagnosis in case of: } \\
\text {-pts with highest risk NMIBC; } \\
\text { - pts with BCG unresponsive tumor or BCG failure. }\end{array}$ \\
\hline $\begin{array}{l}\text { National Comprehensive Cancer Network (NCCN) } \\
\text { (https://www.nccn.org/covid-19/pdf/Cancer_Services_ } \\
\text { Patient_Prioritization_Guidelines.pdf) (11) }\end{array}$ & $\begin{array}{l}\text { Possible postponing low risk pts } \\
\text { Not postponing high risk pts }\end{array}$ & \\
\hline $\begin{array}{l}\text { American Urological Association } \\
\text { (https://www.facs.org//covid-19/clinical-guidance/triage) (12) }\end{array}$ & $\begin{array}{l}\text { High risk: } \\
\text { TURBT as scheduled } \\
\text { Not- high risk: } \\
\text { Postpone in 4-12 weeks }\end{array}$ & $\begin{array}{l}\text { High risk cancer: } \\
\text { Cystectomy as scheduled } \\
\text { Not- high risk cancer: } \\
\text { Postpone in 4-12 weeks }\end{array}$ \\
\hline
\end{tabular}

infections, compared to general population (5, 15). Furthermore, it is important to consider the most valuable resource that is personnel, as we observed a 30\% shortage of health care workers due to hospital-acquired COVID-19 infection $(16,17)$.

We report on the surgical management of $\mathrm{BC}$ in different regions of Italy (with focus on Lombardy, as the Italian epicenter) during the first month of COVID-19 outbreak (March 2020), with head to head comparison with data from March 2019.

\section{MATERIALS AND METHODS}

A survey containing 14 multiple choice questions, focused on surgical activity related to BC carried out in March 2019 and March 2020, was sent to 32 Italian Centers (see appendix 1) during the first week of April 2020. We also queried medical staffing at these facilities: total amount of Urologists (including resident physicians) and percentage dedicated to COVID wards for each institute. 28 Centers answered (Table 2). To note, non COVID-centers (nCC) were defined as hospitals where only pts with negative nasopharyngeal swab and negative chest CT-scans were admitted. Statistical analysis of the data was performed using IBM SPSS Statistics (v26) software. We evaluated the geographic differences between Lombardy (the epicenter of pandemic), Northern centers outside Lombardy, Center and South. Moreover, we evaluated differences between academic (AC) and non-academic centers (NAC), according to location. We evaluated the number of RC, TURBT, operative blocks (OB) dedicated to urology and number of surgical procedures performed during March 2019 and
Table 2.

Main characteristics of centers who answered to survey.

\begin{tabular}{|l|c|}
\hline Characteristic & Number \\
\hline Total amount of centers who answered & $28 / 32(87.5 \%)$ \\
\hline Academic Centers & $15 / 28(53.6 \%)$ \\
\hline Emergency Room & $24 / 28(85.7 \%)$ \\
\hline Non COVID-centers (nCC) & $4 / 28(14.3 \%)$ \\
\hline Institutes located in North & $14 / 28(50 \%)$ \\
\hline Institutes in Lombardy & $11 / 28(39.3 \%)$ \\
\hline Institutes located in Centre & $7 / 28(25 \%)$ \\
\hline Institutes located in South & $7 / 28(25 \%)$ \\
\hline
\end{tabular}

March 2020. We also compared the differences between AC and NAC. All the variables in this survey are nominal (categorical), so we tested the statistical differences between centers and location using chi-square test through crosstab function in our software.

\section{RESULTS}

Table 3 reported data about centers and their distribution across Italy.

Several different statistical differences emerged comparing Lombardy hospitals and rest of Northern Italy in March 2020: number of $O B$ dedicated to urology $(\mathrm{p}=0.027)$; number of surgical procedures per $\mathrm{OB}(\mathrm{p}=0.018)$; number of TURBT ( $\mathrm{p}=0.012$ ); number of hemostatic TransUrethral Resection (hTUR) $(\mathrm{p}=0.010)$. These differences were no relevant considering Lombardy centers among Northern group. 
Table 3.

Lombardy vs North vs Centre and South Italy centers.

\begin{tabular}{|c|c|c|c|c|c|c|}
\hline & Lombardy & North Italy & Central Italy & South Italy & Total numbers & p \\
\hline Number & $11 / 28(39.3)$ & $3 / 28(10.7 \%)$ & $7 / 28(25 \%)$ & $7 / 28(25 \%)$ & & \\
\hline \multicolumn{7}{|l|}{ Covid free } \\
\hline yes & $2 / 11(18.2 \%)$ & $0 / 3$ & $2 / 7(28.6 \%)$ & $0 / 7$ & $4 / 28(14.3 \%)$ & 0.518 \\
\hline no & 9/11 (81.8\%) & $3 / 3$ & $5 / 7(71.4 \%)$ & $7 / 7$ & $24 / 28(85.7 \%)$ & \\
\hline \multicolumn{7}{|c|}{ Academic Hospital } \\
\hline yes & $4 / 11(36.4 \%)$ & 2/3 (66.7\%) & $5 / 7(71.4 \%)$ & $4 / 7(57.1 \%)$ & $15 / 28(53.6 \%)$ & 0.140 \\
\hline no & $7 / 11(63.6 \%)$ & $1 / 3(33.3 \%)$ & $2 / 7(28.6 \%)$ & $3 / 7(42.9 \%)$ & $13 / 28(46.2 \%)$ & \\
\hline \multicolumn{7}{|c|}{ Emergency room: } \\
\hline yes & 9/11 (81.8\%) & $3 / 3$ & $5 / 7(71.4 \%)$ & $7 / 7$ & $24 / 28(85.7 \%)$ & 0.518 \\
\hline no & 2/11 (18.2\%) & $0 / 3$ & $2 / 7(28.6 \%)$ & $0 / 7$ & $4 / 28(14.3 \%)$ & \\
\hline \multicolumn{7}{|l|}{ staff: } \\
\hline$<10$ & $5 / 11(45.5 \%)$ & $1 / 3(33.3 \%)$ & $3 / 7(42.9 \%)$ & $1 / 7(14.3 \%)$ & $10 / 28(35.7 \%)$ & 0.288 \\
\hline $10-20$ & $5 / 11(45.5 \%)$ & $0 / 3$ & $1 / 7(14.3 \%)$ & $5 / 7(71.4 \%)$ & $11 / 28(39.3 \%)$ & \\
\hline$>20$ & $1 / 11(9.1 \%)$ & $2 / 3(66.7 \%)$ & $3 / 7(42.9 \%)$ & $1 / 7(14.3 \%)$ & $7 / 28(25 \%)$ & \\
\hline \multicolumn{7}{|c|}{ Medical Doctors employed in Covid wards: } \\
\hline$<30 \%$ & $5 / 11(45.5 \%)$ & $3 / 3$ & $7 / 7$ & $7 / 7$ & $22 / 28(78.6 \%)$ & 0.003 \\
\hline $30 \%-50 \%$ & $3 / 11(27.3 \%)$ & $0 / 3$ & $0 / 7$ & $0 / 7$ & $3 / 28(10.7 \%)$ & \\
\hline$>50 \%$ & $3 / 11(27.3 \%)$ & $0 / 3$ & $0 / 7$ & $0 / 7$ & $3 / 28(10.7 \%)$ & \\
\hline \multicolumn{7}{|c|}{ Operating block dedicated to Urology Division during March 2020: } \\
\hline 0 & $4 / 11(36.4 \%)$ & $0 / 3$ & $0 / 7$ & $0 / 7$ & $4 / 28(14.3 \%)$ & 0.027 \\
\hline $1-2$ & $1 / 11(9.1 \%)$ & $0 / 3$ & $1 / 7(14.3 \%)$ & $2 / 7(28.6 \%)$ & $4 / 28(14.3 \%)$ & \\
\hline$>2$ & $6 / 11(54.5 \%)$ & $3 / 3$ & $6 / 7(85.7 \%)$ & $5 / 7(71.4 \%)$ & $20 / 28(71.4 \%)$ & \\
\hline \multicolumn{7}{|c|}{ Operating block dedicated to Urology Division during March 2019: } \\
\hline 0 & $0 / 11$ & $0 / 3$ & $0 / 7$ & $0 / 7$ & $0 / 28$ & 0.329 \\
\hline $1-2$ & $1 / 11(9.1 \%)$ & $0 / 3$ & $1 / 7(14.3 \%)$ & $3 / 7(42.9 \%)$ & $5 / 28(17.9 \%)$ & \\
\hline$>2$ & $10 / 11(90.9 \%)$ & $3 / 3$ & $6 / 7(85.7 \%)$ & $4 / 7(57.1 \%)$ & $23 / 28(82.1 \%)$ & \\
\hline \multicolumn{7}{|c|}{ Number of operation performed per operating block during March 2020: } \\
\hline 1 & $4 / 11(36.4 \%)$ & $0 / 3$ & $0 / 7$ & $0 / 7$ & $4 / 28(14.3 \%)$ & 0.018 \\
\hline $2-3$ & $4 / 11(36.4 \%)$ & $3 / 3$ & $5 / 7(71.4 \%)$ & $5 / 7(71.4 \%)$ & $17 / 28(60.7 \%)$ & \\
\hline$>3$ & $3 / 11(27.3 \%)$ & $0 / 3$ & $2 / 7(28.6 \%)$ & $2 / 7(28.6 \%)$ & $7 / 28(25 \%)$ & \\
\hline \multicolumn{7}{|c|}{ Number of operation performed per operating block during March 2019: } \\
\hline 1 & $0 / 11$ & $0 / 3$ & $0 / 7$ & $1 / 7(14.3 \%)$ & $1 / 28(3.6 \%)$ & 0.688 \\
\hline $2-3$ & $7 / 11(63.6 \%)$ & $2 / 3(66.7 \%)$ & $3 / 7(42.9 \%)$ & $6(85.7 \%)$ & $18 / 28(64.3 \%)$ & \\
\hline$>3$ & $4 / 11(36.4 \%)$ & $1 / 3(33.3 \%)$ & $4 / 7(57.1 \%)$ & $0 / 7$ & $9 / 28(32.1 \%)$ & \\
\hline \multicolumn{7}{|c|}{ Number of trans-urethral resection performed during March 2020: } \\
\hline$<5$ & $6 / 11(54.5 \%)$ & $0 / 3$ & $0 / 7$ & $1 / 7(14.3 \%)$ & $7 / 28(25 \%)$ & 0.012 \\
\hline $5-15$ & $4 / 11(36.4 \%)$ & $3 / 3$ & $4 / 7(57.1 \%)$ & $3 / 7(42.9 \%)$ & $14 / 28(50 \%)$ & \\
\hline$>15$ & $3 / 11(27.3 \%)$ & $0 / 3$ & $3 / 7(42.9 \%)$ & $3 / 7(42.9 \%)$ & $7 / 28(25 \%)$ & \\
\hline \multicolumn{7}{|c|}{ Number of trans-urethral resection performed during March 2019: } \\
\hline$<5$ & $0 / 11$ & $0 / 3$ & $0 / 7$ & $1 / 7(14.3 \%)$ & $1 / 28(3.6 \%)$ & 0.664 \\
\hline $5-15$ & $4 / 11(36.4 \%)$ & 1/3 (33.3\%) & $3 / 7(42.9 \%)$ & $3 / 7(42.9 \%)$ & $11 / 28(39.3 \%)$ & \\
\hline$>15$ & $7 / 11(63.6 \%)$ & $2 / 3(66.7 \%)$ & $4 / 7(57.1 \%)$ & $3 / 7(42.9 \%)$ & $16 / 28(57.1 \%)$ & \\
\hline \multicolumn{7}{|c|}{ Number of cystectomy performed during March 2020: } \\
\hline 0 & $6 / 11(54.5 \%)$ & $0 / 3$ & $0 / 7$ & $3 / 7(42.9 \%)$ & $9 / 28(32.1 \%)$ & 0.123 \\
\hline $1-5$ & $3 / 11(27.3 \%)$ & $2 / 3(66.7 \%)$ & $4 / 7(57.1 \%)$ & $3 / 7(42.9 \%)$ & $12 / 28(42.9 \%)$ & \\
\hline$>5$ & $2 / 11(18.2 \%)$ & $1 / 3(33.3 \%)$ & $3 / 7(42.9 \%)$ & $1 / 7(14.3 \%)$ & $7 / 28(25 \%)$ & \\
\hline$\overline{\text { Number of } \mathrm{c}}$ & ystectomy perform & med during March & 2019: & & & \\
\hline 0 & $4 / 11(36.4 \%)$ & $0 / 3$ & $2 / 7(28.6 \%)$ & $6 / 7(85.7 \%)$ & $12 / 28(42.9 \%)$ & 0.688 \\
\hline $1-5$ & $2 / 11(18.2 \%)$ & $1 / 3(33.3 \%)$ & $2 / 7(28.6 \%)$ & $1 / 7(14.3 \%)$ & $6 / 28(21.4 \%)$ & \\
\hline$>5$ & $5 / 11(45.5 \%)$ & $2 / 3(66.7 \%)$ & $3 / 7(42.9 \%)$ & $0 / 7$ & $10 / 28(35.7 \%)$ & \\
\hline Number of $p$ & atients evaluated & for hematuria du & uring March 2020: & & & \\
\hline$<5$ & $7 / 11(63.6 \%)$ & $0 / 3$ & $1 / 7(14.3 \%)$ & $3 / 7(42.9 \%)$ & $11 / 28(39.3 \%)$ & 0.072 \\
\hline $5-10$ & $1 / 11(9.1 \%)$ & $2 / 3(66.7 \%)$ & $2 / 7(28.6 \%)$ & $3 / 7(42.9 \%)$ & $8 / 28(28.6 \%)$ & \\
\hline$>10$ & $3 / 11$ (27.3\%) & $1 / 3(33.3 \%)$ & $4 / 7(57.1 \%)$ & $1 / 7(14.3 \%)$ & $9 / 28(32.1 \%)$ & \\
\hline Number of $p$ & atients evaluated & for hematuria du & uring March 2019: & & & \\
\hline$<5$ & $2 / 11(18.2 \%)$ & $0 / 3$ & $2 / 7(28.6 \%)$ & $2 / 7(28.6 \%)$ & $6 / 28(21.4 \%)$ & 0.937 \\
\hline $5-10$ & $3 / 11(27.3 \%)$ & $0 / 3$ & $1 / 7(14.3 \%)$ & $3 / 7(42.9 \%)$ & $7 / 28(25 \%)$ & \\
\hline$>10$ & $6 / 11(54.5 \%)$ & $3 / 3$ & $4 / 7(57.1 \%)$ & $2 / 7(28.6 \%)$ & $15 / 28(53.6 \%)$ & \\
\hline$\overline{\text { Number of } p}$ & atients undergone & e to tur for hen & static/diagnostic & urpose during $\mathrm{M}$ & |arch 2020: & \\
\hline 0 & $7 / 11(63.6 \%)$ & $0 / 3$ & $1 / 7(14.3 \%)$ & $1 / 7(14.3 \%)$ & $9 / 28(32.1 \%)$ & 0.010 \\
\hline$<50 \%$ & $4 / 11(36.4 \%)$ & $3 / 3$ & $4 / 7(57.1 \%)$ & $4 / 7(57.1 \%)$ & $15 / 28(53.6 \%)$ & \\
\hline$>50 \%$ & $0 / 11$ & $0 / 3$ & $2 / 7(28.6 \%)$ & $2 / 7(28.6 \%)$ & $4 / 28(14.3 \%)$ & \\
\hline Number of & atients undergone & e to tur for hem & static/diagnostic & rpose during $\mathrm{M}$ & larch 2019: & \\
\hline 0 & $2 / 11(18.2 \%)$ & $0 / 3$ & $2 / 7(28.6 \%)$ & $1 / 7(14.3 \%)$ & $5 / 28$ (17.9\%) & 0.412 \\
\hline$<50 \%$ & $7 / 11(63.6 \%)$ & 1/3 (33.3\%) & $3 / 7(42.9 \%)$ & $3 / 7(42.9 \%)$ & $14 / 28(50 \%)$ & \\
\hline$>50 \%$ & $2 / 11(18.2 \%)$ & $2 / 3(66.7 \%)$ & $2 / 7(28.6 \%)$ & $3 / 7(42.9 \%)$ & $9 / 28(32.1 \%)$ & \\
\hline
\end{tabular}

Compared to other hospitals across Italy during March 2020, Lombardy was the only region where a consistent number of urology staff were reassigned to COVID wards ( $p=0.003)$ and four centers had no OB dedicated to urology $(p=0.027)$.

Table 4 describes differences in surgical activity, as well as patients with hematuria referred to emergency room (ER) in different parts of Italy in March 2019 and March 2020. A statistically significant reduction of the amount of RC in Lombardy ( $p=0.036)$ was seen, as well as an increasing number of RC performed in the South ( $\mathrm{p}=$ 0.030). The total amount of $\mathrm{RC}$ remained the same in 2019 and 2020, as more centers performed these opera-

Table 4.

Comparison of 2019 and 2020 activity in Lombardy, North, Centre and South of Italy.

\begin{tabular}{|c|c|c|c|c|c|c|c|c|}
\hline & Lombardy & p & North & p & Central & p & South & p \\
\hline \multicolumn{9}{|c|}{ Operating block dedicated to Urology Division during March 2020: } \\
\hline 0 & $4 / 11(36.4 \%)$ & & $0 / 3$ & & $0 / 7$ & & $0 / 7$ & \\
\hline $1-2$ & $1 / 11(9.1 \%)$ & & $0 / 3$ & & $1 / 7(14.3 \%)$ & & $2 / 7(28.6 \%)$ & \\
\hline$>2$ & $6 / 11(54.5 \%)$ & 0.382 & $3 / 3$ & N.A. & $6 / 7(85.7 \%)$ & 0.143 & $5 / 7(71.4 \%)$ & 0.714 \\
\hline \multicolumn{9}{|c|}{ Operating block dedicated to Urology Division during March 2019: } \\
\hline 0 & 0/11 & & $0 / 3$ & & $0 / 7$ & & 0/7 & \\
\hline $1-2$ & $1 / 11(9.1 \%)$ & & $0 / 3$ & & $1 / 7(14.3 \%)$ & & $3 / 7(42.9 \%)$ & \\
\hline$>2$ & $10 / 11(90.9 \%)$ & & $3 / 3$ & & $6 / 7(85.7 \%)$ & & $4 / 7(57.1 \%)$ & \\
\hline \multicolumn{9}{|c|}{ Number of operation performed per operating block during March 2020: } \\
\hline 1 & $4 / 11(36.4 \%)$ & & $0 / 3$ & & $0 / 7$ & & $0 / 7$ & \\
\hline $2-3$ & $4 / 11(36.4 \%)$ & & $3 / 3$ & & $5 / 7(71.4 \%)$ & & $5 / 7(71.4 \%)$ & \\
\hline$>3$ & $3 / 11(27.3 \%)$ & 0.441 & $0 / 3$ & N.A. & $2 / 7(28.6 \%)$ & 0.286 & $2 / 7(28.6 \%)$ & 0.286 \\
\hline \multicolumn{9}{|c|}{ Number of operation performed per operating block during March 2019: } \\
\hline 1 & 0/11 & & $0 / 3$ & & $0 / 7$ & & $1 / 7(14.3 \%)$ & \\
\hline $2-3$ & $7 / 11(63.6 \%)$ & & $2 / 3(66.7 \%)$ & & $3 / 7(42.9 \%)$ & & $6(85.7 \%)$ & \\
\hline$>3$ & $4 / 11(36.4 \%)$ & & $1 / 3(33.3 \%)$ & & $4 / 7(57.1 \%)$ & & $0 / 7$ & \\
\hline \multicolumn{9}{|c|}{ Number of trans-urethral resection performed during March 2020: } \\
\hline$<5$ & $6 / 11(54.5 \%)$ & & $0 / 3$ & & $0 / 7$ & & $1 / 7(14.3 \%)$ & \\
\hline $5-15$ & $4 / 11(36.4 \%)$ & & $3 / 3$ & & $4 / 7(57.1 \%)$ & & $3 / 7(42.9 \%)$ & \\
\hline$>15$ & $3 / 11(27.3 \%)$ & 0.125 & $0 / 3$ & N.A. & $3 / 7(42.9 \%)$ & 0.629 & $3 / 7(42.9 \%)$ & 0.629 \\
\hline \multicolumn{9}{|c|}{ Number of trans-urethral resection performed during March 2019: } \\
\hline$<5$ & $0 / 11$ & & $0 / 3$ & & $0 / 7$ & & $1 / 7(14.3 \%)$ & \\
\hline $5-15$ & $4 / 11(36.4 \%)$ & & $1 / 3(33.3 \%)$ & & $3 / 7(42.9 \%)$ & & $3 / 7(42.9 \%)$ & \\
\hline$>15$ & $7 / 11(63.6 \%)$ & & $2 / 3(66.7 \%)$ & & $4 / 7(57.1 \%)$ & & $3 / 7(42.9 \%)$ & \\
\hline \multicolumn{9}{|c|}{ Number of cystectomy performed during March 2020: } \\
\hline 0 & $6 / 11(54.5 \%)$ & & $0 / 3$ & & $3 / 7(42.9 \%)$ & & $9 / 28(32.1 \%)$ & \\
\hline $1-5$ & $3 / 11(27.3 \%)$ & & $2 / 3(66.7 \%)$ & & $3 / 7(42.9 \%)$ & & $12 / 28(42.9 \%)$ & \\
\hline$>5$ & $2 / 11(18.2 \%)$ & 0.036 & $1 / 3(33.3 \%)$ & 0.667 & $1 / 7(14.3 \%)$ & 0.327 & $7 / 28(25 \%)$ & 0.030 \\
\hline \multicolumn{9}{|c|}{ Number of cystectomy performed during March 2019: } \\
\hline 0 & $4 / 11(36.4 \%)$ & & $0 / 3$ & & $6 / 7(85.7 \%)$ & & $12 / 28(42.9 \%)$ & \\
\hline $1-5$ & $2 / 11(18.2 \%)$ & & $1 / 3(33.3 \%)$ & & $1 / 7(14.3 \%)$ & & $6 / 28(21.4 \%)$ & \\
\hline$>5$ & $5 / 11(45.5 \%)$ & & $2 / 3(66.7 \%)$ & & $0 / 7$ & & $10 / 28(35.7 \%)$ & \\
\hline \multicolumn{9}{|c|}{ Number of patients evaluated for hematuria during March 2020: } \\
\hline$<5$ & $7 / 11(63.6 \%)$ & & $0 / 3$ & & $1 / 7(14.3 \%)$ & & $3 / 7(42.9 \%)$ & \\
\hline $5-10$ & $1 / 11(9.1 \%)$ & & $2 / 3(66.7 \%)$ & & $2 / 7(28.6 \%)$ & & $3 / 7(42.9 \%)$ & \\
\hline$>10$ & $3 / 11(27.3 \%)$ & 0.264 & $1 / 3(33.3 \%)$ & N.A. & $4 / 7(57.1 \%)$ & 0.068 & $1 / 7(14.3 \%)$ & 0.421 \\
\hline \multicolumn{9}{|c|}{ Number of patients evaluated for hematuria during March 2019: } \\
\hline$<5$ & $2 / 11(18.2 \%)$ & & $0 / 3$ & & $2 / 7(28.6 \%)$ & & $2 / 7(28.6 \%)$ & \\
\hline $5-10$ & $3 / 11(27.3 \%)$ & & $0 / 3$ & & $1 / 7(14.3 \%)$ & & $3 / 7(42.9 \%)$ & \\
\hline$>10$ & $6 / 11(54.5 \%)$ & & $3 / 3$ & & $4 / 7(57.1 \%)$ & & $2 / 7(28.6 \%)$ & \\
\hline \multicolumn{9}{|c|}{ Number of patients undergone to tur for hemostatic/diagnostic purpose during March 2020: } \\
\hline 0 & $7 / 11(63.6 \%)$ & & $0 / 3$ & & $1 / 7(14.3 \%)$ & & $1 / 7(14.3 \%)$ & \\
\hline$<50 \%$ & $4 / 11(36.4 \%)$ & & $3 / 3$ & & $4 / 7(57.1 \%)$ & & $4 / 7(57.1 \%)$ & \\
\hline$>50 \%$ & 0/11 & 0.166 & $0 / 3$ & N.A. & $2 / 7(28.6 \%)$ & 0.190 & $2 / 7(28.6 \%)$ & 0.033 \\
\hline \multicolumn{9}{|c|}{ Number of patients undergone to tur for hemostatic/diagnostic purpose during March 2019: } \\
\hline 0 & $2 / 11(18.2 \%)$ & & $0 / 3$ & & $2 / 7(28.6 \%)$ & & $1 / 7(14.3 \%)$ & \\
\hline$<50 \%$ & $7 / 11(63.6 \%)$ & & $1 / 3(33.3 \%)$ & & $3 / 7(42.9 \%)$ & & $3 / 7(42.9 \%)$ & \\
\hline$>50 \%$ & $2 / 11(18.2 \%)$ & & $2 / 3(66.7 \%)$ & & $2 / 7(28.6 \%)$ & & $3 / 7(42.9 \%)$ & \\
\hline
\end{tabular}


tions in 2020, due to travel restrictions. Some statistically significant difference was observed comparing AC and NAC, with number of medical doctors, including residents, employed in COVID wards being greater in AC $(\mathrm{p}=0.001)$. To note, the number of TURBT $(\mathrm{p}=0.046)$ and number of RC was superior in AC in March 2020 $(p=0.037)$. Moreover, the AC differed in the number of interventions performed per $\mathrm{OB}(\mathrm{p}=0.015)$ and number of hTUR ( $p=0.014$ ), in favor of 2019. On the other hand, in NAC we did not observe any statistically differences in term of surgical performance from 2019 to 2020 , except for the number of TURBT, which was higher in March 2019 ( $\mathrm{p}=0.022$ ).

Finally, we tested the differences between AC and NAC in the different parts of Italy. In Lombardy, we did not find any statistical differences between $\mathrm{AC}$ and NAC in 2019 and in 2020 regarding: number of OB per week, number of interventions performed per $\mathrm{OB}$, number of TURBT nor number of RC. We did not observe any differences in Lombardy between AC and NAC about patients referred to ER with hematuria requiring hTUR during March 2020 and 2019.

We did not find any statistical differences regarding the aforementioned parameters in the other three centers in the North (outside Lombardy).

In the Center regions, we saw statistical differences only in number of pts evaluated for hematuria $(\mathrm{p}=0.030)$ and number of hTUR in 2019 ( $p=0.030)$, with higher numbers performed in AC. Of note, 2 NAC included in this survey did not have emergency rooms.

Finally, there was no statistical differences in the aforementioned items in the Southern regions.

\section{Discussion}

\section{General performance of Italian centers during the first month of Pandemic}

In Italy, from February, $27^{\text {th }}$ to April $28^{\text {th }}, 199,470$ cases and 25.215 confirmed COVID-19-related deaths were reported (18). At the same time, a significant shortage of health care personnel was observed, with 20.831 health workers $(10 \%)$ being affected by confirmed COVID-19 infection $(5,15,18,19)$.

The rapidly increasing number of pts affected by the SARS-Cov-2 virus have exerted significant pressure on the healthcare systems of Western countries in general, with an emphasis on maintaining emergency and essential services. The need to dedicate major economic, infrastructural and health care resources to assist SARS-Cov2 patients during the first weeks of the outbreak resulted in a rapid reallocation of staff, wards and equipment from several medical disciplines not primarily involved in the management of these pts $(5,20)$.

As a result, several facilities had to retrain or re-assign personnel to COVID-Related Activities (CRA), even if this was outside of their primary designation. The majority of hospital wards were converted to COVID-dedicated units and surgical were cases reduced because personnel and resources were reassigned.

In our study, all centers maintained $70 \%$ of their urological staff for "usual" urological activities and only 30\% were reassigned for CRA. Overall more physicians at AC, including residents, were assigned to COVID wards ( $p=$ 0.001). It is important underlying that, in the global emergency scenario caused by COVID-19 pandemic, the Urology residents' training has been critically affected (especially for residents attending the final year of training), with a significant proportion of residents experiencing a severe reduction (>40\%) or complete suppression (> 80\%) of training both for "clinical" activities and "surgical" activities, as reported by Amparore et al. (21). While this involvement was particularly evident in Lombardy, with Urologists involved in CRA in more than $50 \%$ of the hospitals, in the Centre and Southern Italy, $<30 \%$ of Urologists were assigned to CRA.

We can therefore hypothesize that the greater the number of physicians on staff during the usual activity period, the greater the number assigned to CRA during the pandemic.

The data of our survey supports the findings of Naspro (8) and Montorsi (22), from Giovanni XXIII Hospital in Bergamo and San Raffaele Hospital in Milan, respectively (two of the primary centers for COVID-19 management during the pandemic). Naspro reported that, during the 10 days of the first cases of SARS-Cov2, two-thirds of the hospital beds were occupied by pts with COVID-19. Within two weeks, urological surgical volume was reduced to $30 \%$, then $15 \%$ and then totally halted as of March 19. With the progression of the outbreak, all nonemergent urology surgeries were cancelled, with few exceptions for emergent and some urgent cases (8).

During our investigation we identified four non-COVID centers (nCC): two in the North and two in the Center. As expected, the number of urological procedures in nCC was almost identical to that of the same period in 2019. Referring pts to high-volume centers and surgeons potentially allows fast discharge and reduced number of complications $(14,23,24)$. This organization also allowed for the residency program to proceed without interruptions in teaching program $(25,26)$.

There were no nCC in the South in our Survey, but we know that they have been created. This may be partly justified by the reduced number of COVID pts in Southern Italy (18).

Except in the South, where numbers were mildly increased, the total amount of surgical procedures were comprehensively reduced during 2020, with a wider geographic distribution of urological procedures. These is likely a direct result of strict travel restrictions during pandemic, which prevented patients living in the South from seeking medical care in the North of Italy, which was prevoiusly the norm.

A structural reorganization is essential during this time, as key elements, such as the duration of emergency, economic and social consequences, or the viral persistence in the population, are unknown (5). Therefore, rationing resources becomes mandatory, in order to ensure continuity of healthcare for COVID-free patients (27). Nevertheless, the Italian situation has to be considered as peculiar: preservation of COVID-19-free areas within mixed facilities turned out to be impossible: both caregivers and pts can bring the infection while asymptomatic, contributing to further nosocomial spread. 


\section{Overview on surgical management of bladder cancer during pandemic}

When the COVID-19 outbreak expanded into Western Countries during the last weeks of February 2020, there were no recommendations about management of oncological surgical procedures, including urology. During the following weeks, several International and National Scientific Societies have published suggestions based on experts' opinions, using limited data available and with currently unknown impact on urologic practice (Table 1) (9-12).

Campi et al. recently found that approximately two thirds of pts with genitourinary malignancies do not require high-priority surgery, and $25 \%$ of pts requiring high-priority surgery are considered at high perioperative risk. This increasing risk is partially defined by the immunocompromised cancer-related state, which leads to increased susceptibility to infectious diseases compared to general population (15). During this pandemic, the risk of COVID-19 related complications, including ICU admissions, requiring mechanical ventilation and death, has been calculated to be 3.5 folds higher than usual (28). Conversely, Wang et al. have highlighted the risk for cancer pts who do not receive adequate and timely medical treatments during an outbreak, resulting in a potentially dangerous delay of uro-oncologic surgeries, with a final impact on the short and intermediate-term progression and mortality rates $(19,29,30)$.

\section{Trans-urethral resection of bladder tumor}

According to the aforementioned Recommendations, TURBT for Low Grade Non Muscle Invasive Bladder Cancer (LG NMIBC) can be delayed in maximum 6 months during the COVID-19 outbreak. In case of High Grade (HG) NMIBC, the recommendations of all Societies advise against postponing interventions, due to the risk progression to muscle invasion/metastases in $15-40 \%$ and the Cancer Specific Mortality of around 10-20\% $(30,31)$. In case of re-resection, the indications should be carefully evaluated, considering COVID-19 local incidence, patients' risk factors, BC risk, characteristics of initial TURBT, not forgetting the limited surgical capacity during pandemic.

The potential risk of stage migration due to postponing TURBT should always be taken into consideration.

Finally, most NMIBC patients should be considered at high risk of presenting with severe forms of COVID-19 that might require admission to an ICU and invasive ventilation. In this particular context, the urologist has a responsibility to evaluate the potential benefits and risks of performing TURBT at the time (32). Naturally, our results showed a decrease in total amount of TURBTs in March 2020 when compared to March 2019, especially in NAC ( $\mathrm{p}=0.022)$.

These data agree with those recently published by Oderda et al. who reported a a restriction for TURBT of about $46 \%(15,33)$. To note, the number of TURBT performed $(\mathrm{p}=0.046)$ was superior in AC in $2020(\mathrm{p}=$ 0.037 ), probably due to superior number of medical staff and/or the presence of residents. Unfortunately, we did not collect detailed data about TURBT; thus, we cannot comment on this specific issue.
Radical cystectomy

RC should be prioritized to other urologic oncology procedures and never be postponed according to all recommendations during the crisis (Table 1). Delays exceeding 90 days between diagnosis/TURBT and RC are associated with worse survival $(7,34)$.

The intervention should be considered in patients at low risk of COVID-19 mortality and with high-risk disease features: presence of high-grade pTl plus Tis, or tumors with lympho-vascular invasion, variant histology (eg, micro-papillary disease), residual grade 3/high-grade urothelial carcinoma on re-resection, or pTl stage (3537). The total amount of RC was decreased during the pandemic in Italy, consistent with a reduction of $46 \%$ in major uro-oncological surgeries across Europe.

Nevertheless, RC remained the second most common procedure performed during the COVID-19 outbreak (11.7\% of all urological procedures) $(15,33)$.

In our survey, more centers performed this operations in 2020 vs 2019, with an increase in geographic availability of RC across Italy. To note, the number or RC performed in the South Centers during March 2020 did not decrease comparing to 2019; in common times Italian pts move routinely from the Southern regions to the North to address medical needs, as aforementioned. This trend was impacted by travel restrictions during outbreak, leading especially AC to perform RC.

\section{Surgical management of emergency room accesses due to hematuria}

The total amount of pts with hematuria and hTUR have generally decreased during COVID-19 outbreak, perhaps attributable to avoidance of the ER during the pandemic. In fact, while all the hospitals have performed hTUR in < 50\% of cases during both 2019 and 2020, during 2019 more centers performed hTUR in up to $50 \%$ of cases. Considering differences in Centres, there was a difference in the number of pts evaluated for hematuria $(\mathrm{p}=0.030)$ and number of hTUR in 2019 (p $=0.030$ ) in favor of AC. Of note, two centers in this area did not have emergency rooms.

The number of hTUR increased only in the South centers and these data can be explained by the access to ER only in case of life-threatening hematuria and by travel restrictions. All these data can be justified by the general reduction of ER admissions because of non-COVID reasons.

\section{Lombardy overview}

During pandemic, most of the centers were dedicated to the management of COVID-19 and had an ER with medicals staff involved in CRA in more than 50\% of the hospitals ( $\mathrm{p}=0.003$ ). Consequently, the number of urological OB diminished, also considering that four centers had not any $\mathrm{OB}$ dedicated to urology, comparing to other hospitals across Italy $(p=0.027)$. On the other hand, there were more than two OB per week dedicated to urology in the most of the hospitals. However, the total amount of TURBT and RC diminished, similarly to the rest of Italy, even if these operations were more homogeneously distributed across the region, and this is demonstrated by the fact the number of centers which performed from 1 to 5 procedures increased. 


\section{Conclusions}

The survey data supports the findings of the most recent papers, showing a global reduction in number of $\mathrm{BC}$ surgical procedures due to the prioritization given to COVID19 pts management. However, we can affirm that the reduction was not so significant if we look at the different regions, especially the Southern ones. In fact, during pandemic, patients seek care at the nearest medical institution, not only for COVID-19 related reasons, overriding the strict travel limitations and leading to an improvement of "local" BC surgical management in the South.

Altogether, these data demonstrate the significant efforts were made by Italian Urologists to proceed with urgent surgical procedures despite COVID-19 outbreak. In our opinion, the improvement of management of pts may be optimized by having COVID-dedicated hospitals to guarantee high-quality, timely, and safe treatments to oncological patients. This leads to appropriate cure both for COVID and COVID-free pts who are affected with urologic cancer and should not have a delay in definitive management. The "overlapping" of COVID- and nonCOVID wards could not ameliorate the management of all pts, because of the risk of transmitting infections by both pts themselves and medical/nursing staff, despite all the strict preventive measures.

Finally, COVID-19 pandemic represents an important challenge and learning opportunity for cancer centers, in the context of an extremely dynamic clinical and political situation which requires maximum flexibility to be appropriately faced. For example, telemedicine can represent an alternative for both multidisciplinary and follow-up visits, as suggested by the preliminary experience of Ambrosini et al. (38).

Our real-life data from several centers across Italy, despite limited, may represent an important insight into the $\mathrm{BC}$ surgical management in times of emergency, giving food for thought about the near future, which will likely be characterized by a prolonged coexistence with SARS-Cov-2 epidemic all over the world.

\section{REFERENCES}

1. Cumberbatch MGK, Jubber I, Black PC, et al. Epidemiology of bladder cancer: a systematic review and contemporary update of risk factors in 2018. Eur Urol. 2018; 74:784-95.

2. Miller KD, Nogueira L, Mariotto AB, et al. Cancer treatment and survivorship statistics, 2019. CA Cancer J Clin. 2019; 69:363-85.

3. Goossens-Laan CA, Leliveld AM, Verhoeven RH, et al. Effects of age and comorbidity on treatment and survival of patients with muscle-invasive bladder cancer. Int J Cancer. 2014; 135:905-12.

4. Noon AP, Albertsen PC, Thomas F, et al. Competing mortality in patients diagnosed with bladder cancer: evidence of undertreatment in the elderly and female patients. Br J Cancer 2013; 108:1534-40.

5. Ficarra V, Novara G, Abrate A, et al. Urology practice during COVID-19 pandemic. Minerva Urol Nefrol. In press. https://doi. org/10.23736/S0393-2249.20.03846-1.

6. Decree Law n 6, 23 February 2020 - "Urgent measures to contain and manage the epidemiological emergency due to COVID-19". https://www.gazzettaufficiale.it/eli/id/2020/02/23/20G00020/sg.

7. Wallis CJD, Novara G, Marandino L, et al. Risks from deferring treatment for genitourinary cancers: a collaborative review to aid triage and management during the COVID-19 pandemic. Eur Urol. 2020; 78:29-42.

8. Naspro R, Da Pozzo L. Urology in the time of corona. Nat Rev Urol. 2020; 17:251-253.

9. https://www.siu.it

10. https://uroweb.org/wp-content/uploads/EAU-Guidelines-OfficeRapid-Reaction-Group-An-organisation-wide-collaborative-effortto-adapt-the-EAU-guidelines-recommendations-to-the-COVID-19era.pdf.

11. https://www.nccn.org/covid19/pdf/Cancer_Services_Patient_ Prioritization_Guidelines.pdf

12. https://www.facs.org/covid-19/clinical-guidance/triage

13. Liang W, Guan W, Chen R, et al. Cancer patients in SARS-CoV2 infection: a nationwide analysis in China. Lancet Oncol. 2020; 21:335-7.

14. Moschovas MC, Sighinolfi MC, Rocco B, et al. Balancing the effects of COVID-19 against potential progression and mortality in high-risk prostate cancer. Eur Urol. 2020; 78:e14-e15.

15. Campi R, Amparore D, Capitanio U, et al. Assessing the burden of nondeferrable major uro-oncologic surgery to guide prioritisation strategies during the COVID-19 pandemic: insights from three Italian high-volume referral centres. Eur Urol. 2020; 78:11-15.

16. Shared protocol for the regulation of measures to contrast and contain the diffusion of COVID-19 virus in the workplace, 14th March 2020. http://www.governo.it/sites/new.governo.it/files/protocollo_condiviso_20200314.pdf.

17. Ti LK, Ang LS, Foong TW, et al. What we do when a COVID-19 patient needs an operation: operating room preparation and guidance. Can J Anaesth. 2020; 67:756-758.

18. https://www.epicentro.iss.it/coronavirus/bollettino/Infografica_ 19 marzo\%20ENG.pdf

19. Stensland KD, Morgan TM, Moinzadeh A, et al. Considerations in the triage of urologic surgeries during the COVID-19 pandemic. Eur Urol. 2020; 77:663-666.

20. Goldman HB, Haber GP. Recommendations for tiered stratification of urological surgery urgency in the COVID-19 era. J Urol. 2020; 204:11-13.

21. Amparore D, Claps F, Cacciamani GE, et al. Impact of the COVID-19 pandemic on urology residency training in Italy. Minerva Urol Nefrol. 2020 Apr 7. doi: 10.23736/S03932249.20.03868-0. Online ahead of print.PMID: 32253371.

22. Montorsi F. We should not ignore what scientific articles are telling us: a lesson from the Italian COVID-19 experience. J Urol. 2020; 204:5-6.

23. Campi R, Zeng G, Dasgupta P, 16 March 2020. https://www. bjuinternational.com/bjui-blog/covid-19-collection-of-urologypapers/.

24. Lei S, Jiang F, Su W, et al. Clinical characteristics and outcomes of patients undergoing surgeries during the incubation period of COVID-19 infection EClinicalMedicine. 2020; 21:100331.

25. Gabrielson AT, Kohn TP, Clifton MM. COVID-19 and the Urology Match: Perspectives and a Call to Action. J Urol. 2020; 204:17-19.

26. Vargo E, Ali M, Henry F, Kmetz D, et al. Cleveland Clinic Akron General Urology Residency Program's COVID-19 Experience. Urology. 2020; 140:1-3. 
27. Leonardi R, Bellinzoni P, Broglia L, et al. Hospital care in Departments defined as Covid-free: A proposal for a safe hospitalization protecting healthcare professionals and patients not affected by Covid 19. Arch Ital Urol Androl. 2020; 92:67-72.

28. Puliatti S, Eissa A, Eissa R, et al. COVID-19 and Urology: a comprehensive review of the literature. BJU Int. 2020; 125:E7-E14.

29. Wang H, Zhang L. Risk of COVID-19 for patients with cancer. Lancet Oncol. 2020; 21:e181.

30. Klaassen Z, Kamat AM, Kassouf W, et al. Treatment strategy for newly diagnosed $T 1$ high-grade bladder urothelial carcinoma: new insights and updated recommendations. Eur Urol. 2018; 74: $597-$ 608.

31. Thomas F, Noon AP, Rubin N, et al. Comparative outcomes of primary, recurrent, and progressive high-risk non-muscle-invasive bladder cancer. Eur Urol. 2013; 63:145-54.

32. Lenfant $L$, Seisen T, Loriot $Y$, Rouprêt M. Adjustments in the use of intravesical instillations of Bacillus Calmette-Guérin for high-risk non-muscle-invasive bladder cancer during the COVID-19 pandemic. Eur Urol. 2020; pii: S0302-2838(20)30302-X.

33. Oderda M, Roupret M, Marra G, et al.The Impact of COVID19 outbreak on uro-oncological practice across Europe: which burden of activity are we facing ahead? Eur Urol. 2020; pii: S03022838(20)30299-2.

\section{Correspondence}

Carmen Maccagnano, MD, FEBU (Corresponding Author)

carmen.maccagnano@gmail.com

Giario Natale Conti, MD

giario.conti@gmail.com

Department of Surgery - Section of Urology

ASST Lariana, Nuovo Ospedale Sant'Anna

Via Ravona, 20, 22042 San Fermo della Battaglia (CO)

Lorenzo Rocchini, MD

lorenzo.rocchini@gmail.com

Department of Surgery - Division of Urology

Emanuele Montanari, MD, Professor of Urology

emanuele.montanari@unimi.it

Department of Surgery - Division of Urology and Department of Clinical

Sciences and Community Health

Fondazione IRCCS Ca' Granda - Ospedale Maggiore Policlinico

Padiglione Cesarina Riva - Via della Commenda 15 - 20122 Milan, Italy

Giovanni Petralia, MD

giovannipetralia@hotmail.com

Urology Unit, Niguarda Hospital

Piazza Ospedale Maggiore, 3 20132, Milan, Italy

Federico Dehò, MD

federico.deho@asst-settelaghi.it

Unit of Urology; ASST Sette Laghi-Circolo e Fondazione Macchi Hospital

Viale Luigi Borri, 57 - 1100 Varese, Italy

Kadi-Ann Bryan, MD

kadiann.bryan@gmail.com

Rogue-Valley Urology - Providence Plaza, Suite 280

1698 E. McAndrews Road - Medford, Oregon 97504

Roberto Contieri, MD

contieri.ro@gmail.com

Rodolfo Hurle, MD

rodolfo.hurle@humanitas.it

Department of Urology - Istituto Clinico Humanitas IRCCS

Clinical and Research Hospital

Via Manzoni, 56 - 20089, Rozzano (MI), Italy
34. Ueda M, Martins R, Hendrie PC, et al. Managing cancer care during the COVID-19 pandemic: agility and collaboration toward a common goal. J Natl Compr Canc Netw. 2020:1-4.

35. Sylvester RJ, van der Meijden AP, Oosterlinck W, et al. Predicting recurrence and progression in individual patients with stage Ta T1 bladder cancer using EORTC risk tables: a combined analysis of 2596 patients from seven EORTC trials. Eur Urol. 2006; 49:466-5; discussion 475-7.

36. Gontero P, Sylvester R, Pisano F, et al. Prognostic factors and risk groups in T1G3 non-muscle-invasive bladder cancer patients initially treated with bacillus Calmette-Guerin: results of a retrospective multicenter study of 2451 patients. Eur Urol. 2015; 67:74-82.

37. Veskimae E, Espinos EL, Bruins HM, et al. What is the prognostic and clinical importance of urothelial and nonurothelial histological variants of bladder cancer in predicting oncological outcomes in patients with muscle-invasive and metastatic bladder cancer? A European Association of Urology Muscle Invasive and Metastatic Bladder Cancer Guidelines Panel Systematic Review. Eur Urol Oncol. 2019; 2 :625-642.

38. Ambrosini F, Di Stasio A, Mantica G, et al. COVID-19 pandemic and uro-oncology follow up: "virtual" multidisciplinary team strategy and patients' satisfaction assessment Arch Ital Urol Androl. 2020; 92:78-79. 